\title{
The Characteristics of New SSB Proteins from Metagenomic Libraries and Their Use in Biotech Applications ${ }^{+}$
}

\author{
Olesia Werbowy 1, Aleksandra Stefańska-Kaźmierczak 2, Agata Jurczak-Kurek ${ }^{3}$, \\ Karolina Kwiatkowska-Semrau ${ }^{1}$, Anna-Karina Kaczorowska ${ }^{4}$, Magdalena Płotka ${ }^{1}$, \\ Olafur H. Fridjonsson 5, Gudmundur O. Hreggvidsson 5, Slawomir Dąbrowski ${ }^{2}$ \\ and Tadeusz Kaczorowski 1,*
}

1 Laboratory of Extremophiles Biology, Department of Microbiology, University of Gdansk, 80-308 Gdansk, Poland; olesia.werbowy@ug.edu.pl (O.W.); karolina.semrau@biol.ug.edu.pl (K.K.-S.); magdalena.plotka@biol.ug.edu.pl (M.P.)

2 A\&A Biotechnology, 81-451 Gdynia, Poland; stefanska@aabiot.com (A.S.-K.); sd@aabiot.com (S.D.)

3 Department of Molecular Evolution, University of Gdansk, 80-308 Gdansk, Poland; a.jurczak-kurek@biol.ug.edu.pl

4 Collection of Plasmids and Microorganisms, University of Gdansk, 80-309 Gdansk, Poland; anna.kaczorowska@ug.edu.pl

5 Matis, 12113 Reykjavik, Iceland; olafur.h.fridjonsson@matis.is (O.H.F.); gudmundur.o.hreggvidsson@matis.is (G.O.H.)

* Correspondence: tadeusz.kaczorowski@biol.ug.edu.pl

† Presented at Viruses 2020-Novel Concepts in Virology, Barcelona, Spain, 5-7 February 2020.

Published: 21 July 2020

\begin{abstract}
Single-stranded DNA binding proteins (SSBs) bind to single-stranded DNA in a sequenceindependent manner to prevent formation of secondary structures and protect DNA from nuclease degradation. These ubiquitous proteins are present in prokaryotes, eukaryotes, and viruses, and play a pivotal role in the following major cellular processes: replication, recombination, and repair of genetic material. In DNA replication, SSB proteins specifically stimulate DNA polymerase, increase fidelity of DNA synthesis, assist the advance of DNA polymerase, and organize and stabilize replication forks. Here, we present our characterization of four SSB proteins of different origins. One of them was isolated from Clostridium sp. phage phiCP130 (SSB C1: $124 \mathrm{aa}, \mathrm{Mr}=13,905$ ). Three others (SSB M2: 136 aa, $\mathrm{Mr}=15,009$; SSB M3: $144 \mathrm{aa}, \mathrm{Mr}=16,106$; and SSB M5: 138 aa, $\mathrm{Mr}=$ $15,851)$ were isolated from metagenomics libraries. They show high similarity to SSB proteins from Caldanaerovirga acetigigens, Caldanaerobius fijiensis, and Fervidobacterium gondwanense. The recombinant proteins were overproduced in E. coli Rosetta (pRARE), except for SSB M5, which was overproduced in E. coli BL21. Proteins were purified using a metal-affinity chromatography as Histagged fusion proteins. Electrophoretic mobility shift assay was used to examine their DNA binding activity with fluorescein-labeled oligonucleotide (dT40) used as a substrate. Thermal stability analysis revealed that they are stable at elevated temperatures, with the exception of SSB protein $\mathrm{C} 1$, which loses its activity above $65^{\circ} \mathrm{C}$. The other proteins are active at high temperatures, SSB M3 up to $85^{\circ} \mathrm{C}$, while SSB M2 and SSB M5 are active up to $98.7^{\circ} \mathrm{C}$. The subunit structure of proteins was analyzed by gel filtration on Superdex 75 column (AKTA). This allowed us to conclude that in solution, the analyzed proteins exist in oligomeric form, a feature which is characteristic of other SSB proteins. Purified SSB proteins were tested to improve specificity of PCR-based DNA amplification.
\end{abstract}


Keywords: DNA-binding protein; single-stranded DNA; oligomers

(9) (1)

(C) 2020 by the authors. Licensee MDPI, Basel, Switzerland. This article is an open access article distributed under the terms and conditions of the Creative Commons Attribution (CC BY) license (http://creativecommons.org/licenses/by/4.0/). 